\title{
Analysis of Rowland Clark Site Corn
}

Leonard Blake

Unknown

Follow this and additional works at: https://scholarworks.sfasu.edu/ita

Part of the American Material Culture Commons, Archaeological Anthropology Commons, Environmental Studies Commons, Other American Studies Commons, Other Arts and Humanities Commons, Other History of Art, Architecture, and Archaeology Commons, and the United States History Commons

Tell us how this article helped you.

This Article is brought to you for free and open access by the Center for Regional Heritage Research at SFA ScholarWorks. It has been accepted for inclusion in Index of Texas Archaeology: Open Access Gray Literature from the Lone Star State by an authorized editor of SFA ScholarWorks. For more information, please contact cdsscholarworks@sfasu.edu. 


\section{Analysis of Rowland Clark Site Corn}

Creative Commons License

(c) () () (9)

This work is licensed under a Creative Commons Attribution-NonCommercial 4.0 International License 


\section{Appendix 1 \\ Analysis of Rowland Clark Site Corn}

\section{Leonard Blake}

The carbonized corn from the Rowland Clark site, Red River County, Texas was received from Gregory Perino, then of the Museum of the Red River, in March 1982. The analysis of the corn is presented by feature, with remarks on the cobs as appropriate. Table 1 presents a summary by feature of the corn cob analysis, while Table 2 compares the corn from Rowland Clark with samples of corn cobs from other well-studied Caddoan sites.

\section{Feature No. 9. Smudge Pit}

\begin{tabular}{|c|c|c|c|c|}
\hline $\mathrm{Cob}$ & Row & Grain & Cupule & $\underline{\text { Remarks }}$ \\
\hline No. & No. & Thickness & Width & \\
\hline 1 & 12 & $2.8 \mathrm{~mm}$ & $5.4 \mathrm{~mm}$ & \\
\hline 2 & 12 & 3.0 & 4.7 & \\
\hline 3 & 12 & 2.9 & 4.9 & \\
\hline 4 & 16 & 2.8 & 4.0 & \\
\hline 5 & 14 & 3.3 & 5.0 & \\
\hline 6 & 12 & 3.2 & 6.2 & \\
\hline 7 & 8 & 3.1 & 5.8 & \\
\hline 8 & 12 & 2.9 & 5.0 & Broken in handling. \\
\hline 9 & 10 & 3.0 & 5.2 & Three segments. \\
\hline 10 & 8 & 2.9 & 6.0 & \\
\hline 11 & 12 & 2.8 & 5.0 & \\
\hline 12 & 10 & 3.4 & 5.0 & \\
\hline 13 & 12 & 3.4 & 5.9 & \\
\hline 14 & 12 & 3.2 & 4.5 & \\
\hline 15 & 10 & 3.1 & 5.8 & Four segments. \\
\hline 16 & 10 & 3.0 & 5.3 (average) & $5.7,5.7,5.0,5.0$, and $5.0 \mathrm{~mm}$. \\
\hline 17 & 12 & 3.2 & 5.6 & \\
\hline 18 & 12 & 3.2 & 5.8 & Tip. \\
\hline 19 & 14 & 3.2 & 5.0 & Five segments broken in handling. \\
\hline 20 & 12 & 3.0 & 5.5 & \\
\hline 21 & 10 & 3.3 & 5.0 & \\
\hline 22 & 12 & 3.4 & 5.0 & \\
\hline 23 & 10 & 3.4 & 5.7 & \\
\hline 24 & 10 & 3.2 & 6.9 & \\
\hline 25 & 12 & 3.7 & 6.3 & \\
\hline 26 & 12 & 3.5 & 5.7 (average) & $5.0,5.0,6.0,6.0,6.0$, and $6.0 \mathrm{~mm}$. \\
\hline 27 & 12 & 3.5 & 6.8 & \\
\hline 28 & 12 & 3.5 & 6.1 & \\
\hline 29 & 14 & 2.5 & 4.6 & \\
\hline 30 & 14 & 2.3 & 5.0 & \\
\hline 31 & 12 & 3.4 & 4.1 & \\
\hline 32 & 10 & 3.5 & 4.9 & \\
\hline 33 & 8 & 3.2 & 5.8 (average) & $5.5,5.5,6.0$, and $6.0 \mathrm{~mm}$. \\
\hline 34 & 12 & 3.6 & 5.3 & \\
\hline
\end{tabular}


Row No.

No. of Cobs

$\%$ of Total

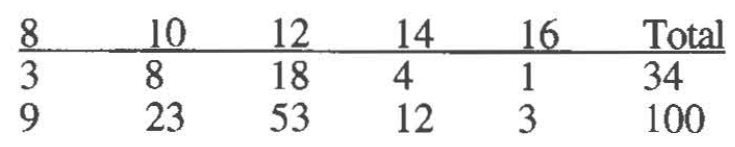

Mean Row No. $=11.5$

Median Cupule Width $=5.3 \mathrm{~mm}$

\section{Feature No. 10. Smudge Pit}

$\underline{\text { Cob }} \underline{\text { Row }}$ Grain $\underline{\text { Cupule }}$ Remarks

No. No. Thickness Width

$\begin{array}{lllll}1 & 16 & 3.8 \mathrm{~mm} & 6.4 \mathrm{~mm} & \\ 2 & 12 & 3.6 & 6.0 & \\ 3 & 10 & 3.4 & 6.6 & \text { Broken in handling. } \\ 4 & 10 & 3.0 & 7.0 & \\ 5 & 10 & 3.6 & 7.4 & \text { Tip end. } \\ 6 & 14 & 3.4 & 4.6 & \text { Tip end. } \\ 7 & 10 & 3.9 & 5.5 & \text { Tip end. } \\ 8 & 12 & 3.5 & 4.9 & \\ 9 & 10 & 3.2 & 6.0 & \\ 10 & 12 & 3.3 & 6.2 & \\ 11 & 12 & 3.7 & 6.5 & \\ 12 & 12 & 3.0 & 5.5 & \\ 13 & 12 & 3.1 & 5.0 & \\ 14 & 10 & 3.9 & 8.0 & \\ 15 & 12 & 3.2 & 5.0 & \\ 16 & 12 & 2.9 & 5.0 & \\ 17 & 12 & 3.6 & 6.6 & \\ 18 & 12 & 3.8 & 5.0 & \\ 19 & 10 & 3.4 & 7.0 & \\ 20 & 12 & 3.2 & 6.5 & \end{array}$

Row No.

No. of Cobs

$\%$ of Total

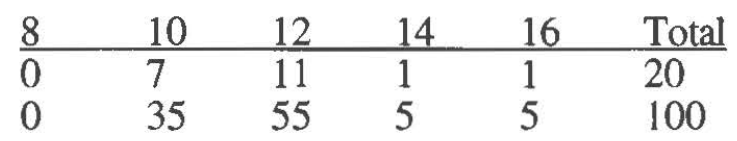

Mean Row No. $=11.6$

Median Cupule Width $=6.1 \mathrm{~mm}$

\section{Feature 14, Smudge Pit}

\begin{tabular}{|c|c|c|c|c|}
\hline Cob & $\underline{\text { Row }}$ & Grain & Cupule & $\underline{\text { Remarks }}$ \\
\hline No. & No. & Thickness & Width & \\
\hline 1 & 10 & $2.5 \mathrm{~mm}$ & $6.5 \mathrm{~mm}$ & \\
\hline 2 & 8 & 3.2 & 8.8 & \\
\hline 3 & 12 & 2.9 & 5.0 & \\
\hline 4 & 12 & 3.1 & 4.8 (average) & four cupules $5.0 \mathrm{~mm}$, two cupules $4.5 \mathrm{~mm}$ \\
\hline 5 & 12 & 3.2 & 6.0 & \\
\hline 6 & 12 & 3.5 & 5.8 & \\
\hline 7 & 12 & 3.4 & 5.8 & \\
\hline 8 & 10 & 3.2 & 7.6 & \\
\hline 9 & 12 & 3.6 & 5.1 & \\
\hline
\end{tabular}




$\begin{array}{lllll}10 & 12 & 3.4 & 4.9 \text { (average) } & \text { cupules } 4.8 \text { to } 5.0 \mathrm{~mm} \\ 11 & 10 & 3.5 & 6.7 & \\ 12 & 12 & 3.0 & 5.5 & \\ 13 & 10 & 3.8 & 6.8 & \\ 14 & 12 & 3.1 & 4.8 & \\ 15 & 10 & 3.1 & 6.2 & \text { Three segments } \\ 16 & 10 & 3.2 & 7.4 & \end{array}$

Row No.

No. of Cobs

$\%$ of Total

\begin{tabular}{llllll}
8 & 10 & 12 & 14 & 16 & Total \\
\hline 1 & 6 & 9 & 0 & 0 & 16 \\
6 & 38 & 56 & 0 & 0 & 100
\end{tabular}

Mean Row No. $=11.0$

Median Cupule Width $=5.9 \mathrm{~mm}$

\section{Feature No. 24. Smudge Pit}

$\begin{array}{llll}\text { Cob } & \text { Row } & \text { Grain } & \text { Cupule } \\ \text { No. } & \underline{\text { Remickness }} & \underline{\text { Width }} & \end{array}$

$\begin{array}{rrlll}1 & 12 & 3.2 \mathrm{~mm} & 5.4 \mathrm{~mm} & \\ 2 & 10 & 3.2 & 4.8 & \\ 3 & 12 & 3.2 & 5.1 & \\ 4 & 8 & 3.2 & 5.8 & \text { Cob section only three cupules long. } \\ 5 & 10 & 3.3 & 5.3 & \\ 6 & 10 & 3.0 & 4.8 & \\ 7 & 10 & 3.0 & 5.6 & \\ 8 & 10 & 3.3 & 6.7 & \\ 9 & 10 & 3.0 & 5.7 \text { (average) } & \text { four cupules } 6.0 \mathrm{~mm} \text {, one } 4.3 \mathrm{~mm} \text { wide. } \\ 10 & 10 & 2.8 & 5.0 & \\ 11 & 12 & 3.0 & 5.0 & \text { Broken in measuring. } \\ 12 & 12 & 3.1 & 4.3 \text { (average) } & \text { four cupules } 4.9 \mathrm{~mm} \text {, two aborted } 3.2 \mathrm{~mm} \\ 13 & 12 & 3.4 & 4.3 \text { (average) } & \text { four cupules } 4.9 \mathrm{~mm} \text {, two aborted } 3.0 \mathrm{~mm} \\ 14 & 8 & 3.0 & 6.4 & \\ 15 & 10 & 3.0 & 4.7 & \\ 16 & 10 & 3.1 & 5.5 & \\ 17 & 10 & 3.4 & 7.0 & \\ 18 & 12 & 3.6 & 7.0 & \\ 19 & 12 & 2.9 & 4.9 & \\ 20 & 10 & 3.0 & 5.2 & \\ 21 & 12 & 3.6 & 5.4 \text { (average) } & \text { four segments, 5.1, 5.1, 5.6, and } 5.6 \mathrm{~mm} \\ 22 & 10 & 2.9 & 5.1 & \text { Broken in handling. } \\ 23 & 12 & 3.6 & 5.5 & \end{array}$

Row No.

No. of Cobs

$\%$ of Total

\begin{tabular}{llllll}
8 & 10 & 12 & 14 & 16 & Total \\
\hline 2 & 12 & 9 & 0 & 0 & 23 \\
9 & 52 & 39 & 0 & 0 & 100
\end{tabular}

Mean Row No. $=10.6$

Median Cupule Width $=5.3 \mathrm{~mm}$ 


\section{Feature No. 25. Smudge Pit}

\begin{tabular}{|c|c|c|c|}
\hline Cob Row & Grain & Cupule & Remarks \\
\hline No. & Thickness & Width & \\
\hline 10 & $3.5 \mathrm{~mm}$ & $5.5 \mathrm{~mm}$ & \\
\hline 10 & & 5.6 & \\
\hline 10 & 3.6 & 5.8 & \\
\hline 12 & 3.0 & 6.0 & Broken in measuring. \\
\hline 12 & 2.7 & 6.0 & \\
\hline 10 & 2.9 & 5.6 & \\
\hline 8 & 3.8 & 7.9 (average) & Two segments, 7.7 and 8.0 cupule width \\
\hline 12 & 5.0 & 7.8 (average) & $7.0,8.0,8.0$, and $8.0 \mathrm{~mm}$ \\
\hline 12 & 3.1 & 4.0 & \\
\hline
\end{tabular}

Row No.

No. of Cobs

$\%$ of Total

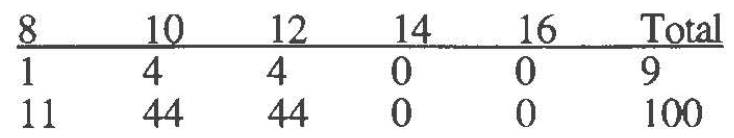

Mean Row No. $=10.6$

Median Cupule Width $=5.5 \mathrm{~mm}$

Feature No. 26, Smudge Pit

\begin{tabular}{|c|c|c|c|}
\hline Row & Grain & Cupule & Remarks \\
\hline$\underline{\text { No. }}$ & Thickness & $\underline{\text { Width }}$ & \\
\hline 8 & $3.5 \mathrm{~mm}$ & $7.8 \mathrm{~mm}$ & \\
\hline 10 & & & \\
\hline 10 & 2.6 & 5.4 & \\
\hline 8 & 2.4 & 8.0 & \\
\hline 10 & 2.4 & 6.0 & \\
\hline 10 & 2.6 & 6.1 & Three segments. \\
\hline 8 & 3.1 & 5.6 & Tip end. \\
\hline 10 & 3.0 & 5.4 & \\
\hline 12 & 2.8 & 4.1 (average) & 3.5-4.8 in six measurements \\
\hline 10 & 3.0 & 5.0 & Broken in measuring. \\
\hline
\end{tabular}

Row No.

No. of Cobs

$\%$ of Total

\begin{tabular}{llllll}
8 & 10 & 12 & 14 & 16 & Total \\
\hline 4 & 5 & 1 & 0 & 0 & 10 \\
40 & 50 & 10 & 0 & 0 & 100
\end{tabular}

Mean Row No. $=9.4$

Median Cupule Width $=5.5 \mathrm{~mm}$

Feature No. 61, Smudge Pit

Cob Row Grain $\underline{\text { Cupule }}$ Remarks

No. No. Thickness Width

$\begin{array}{llll}1 & 12 & 3.8 \mathrm{~mm} & 6.3 \mathrm{~mm}\end{array}$ 
Other cob fragments appear to be parts of the same measured cob. Charred plant materials sent from this feature consisted mostly of wood charcoal and pine cones. A similar smudge pit with pine cones has been examined by Fritz (1992) from 41RR236, a few miles upstream from Rowland Clark.

\section{Feature No. 64}

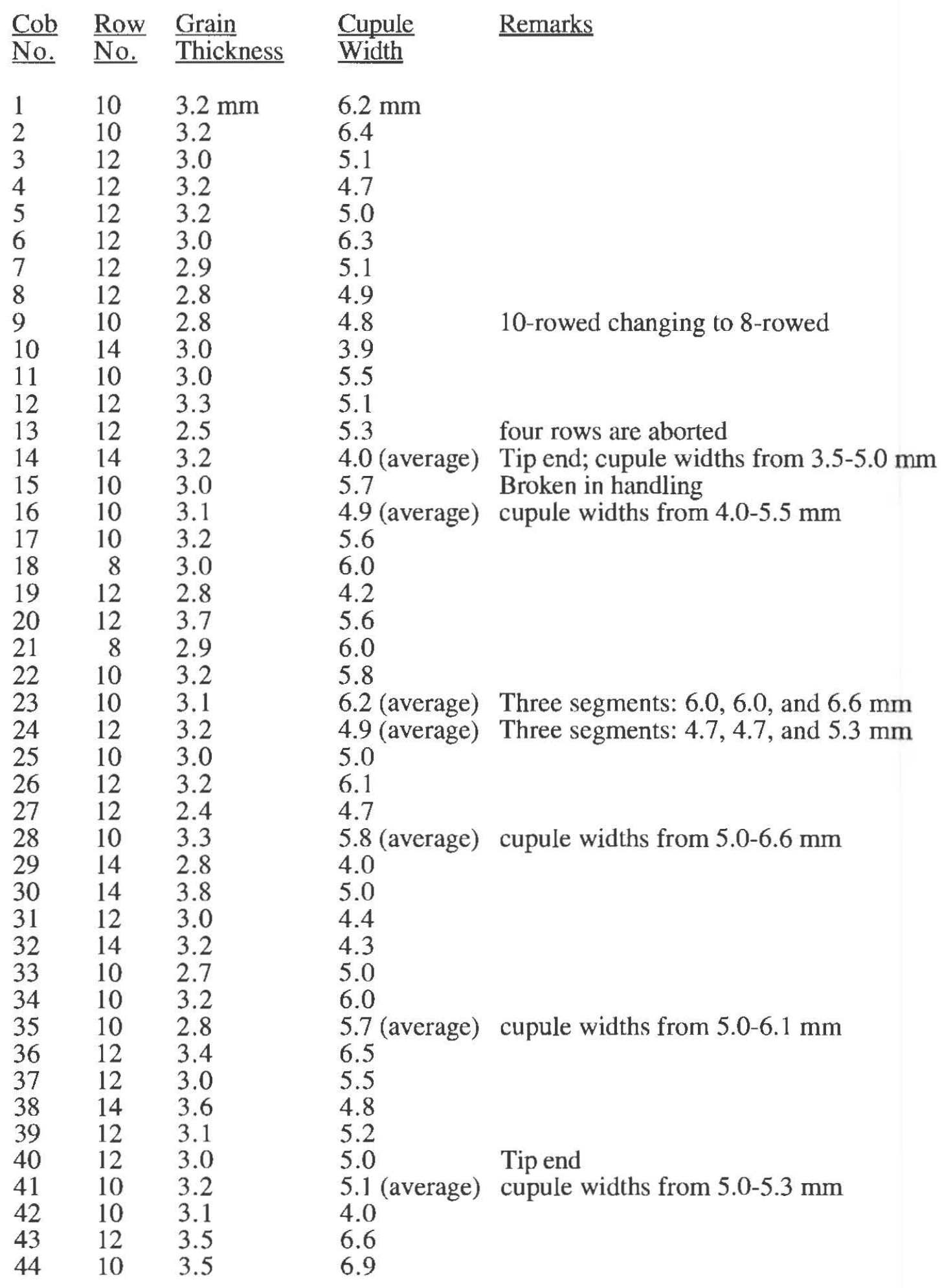




$\begin{array}{rrrrl}45 & 8 & 3.4 & 7.5 & \\ 46 & 14 & 3.6 & 5.3 & \text { Tip end } \\ 47 & 12 & 3.2 & 5.5 & \\ 48 & 12 & 3.6 & 5.0 & \text { Tip end } \\ 49 & 14 & 3.2 & 4.6 & \\ 50 & 8 & 3.1 & 7.8 & \\ 51 & 12 & 2.8 & 4.0 & \text { Tip end } \\ 52 & 10 & 3.8 & 7.0 & \text { 14-rowed changing to 12-rowed } \\ 53 & 12 & 2.6 & 5.4 & \\ 54 & 14 & 3.0 & 3.9 & \\ 55 & 10 & 3.8 & 7.4 & \text { Two segments } \\ 56 & 12 & 3.4 & 6.0 & \text { Three segments } \\ 57 & 10 & 3.1 & 6.3 & \\ 58 & 12 & 3.8 & 6.7 & 5.3\end{array}$

Row No.

No. of Cobs

$\%$ of Total

\begin{tabular}{llllll}
8 & 10 & 12 & 14 & 16 & Total \\
\hline 4 & 20 & 26 & 9 & 0 & 59 \\
7 & 34 & 44 & 15 & 0 & 100
\end{tabular}

Mean Row No. $=11.4$

Median Cupule Width $=5.3 \mathrm{~mm}$

Besides the corn cobs, there were also six pine cones and three pieces of pine cones in Feature 64.

Table 1. Summary of Corn Cob Analysis.

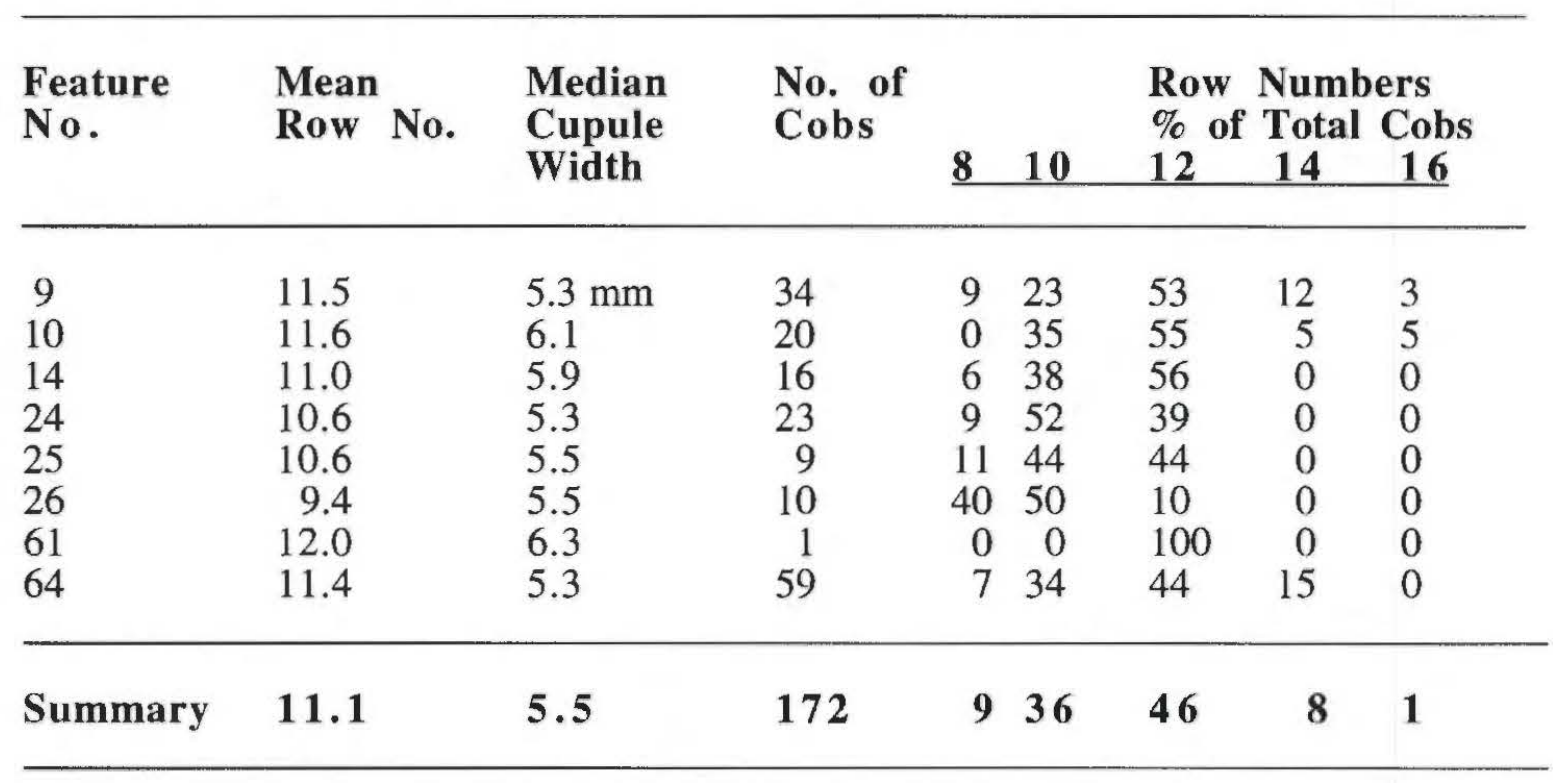


Table 2. Comparison with Corn from other Caddoan Sites.

\begin{tabular}{|c|c|c|c|c|c|c|c|c|}
\hline $\begin{array}{l}\text { Sites and } \\
\text { Age }\end{array}$ & $\begin{array}{l}\text { Mean Row } \\
\text { No. }\end{array}$ & $\begin{array}{l}\text { Median } \\
\text { Cupule } \\
\text { Width }\end{array}$ & $\begin{array}{l}\text { No. of } \\
\text { Cobs }\end{array}$ & $\underline{8}$ & $\begin{array}{l}\text { Rov } \\
\% 0 \\
10 \\
\end{array}$ & $\begin{array}{l}\text { Num } \\
\text { Tota } \\
12 \\
\end{array}$ & $\begin{array}{l}\text { ers } \\
\text { Cobs } \\
14 \\
\end{array}$ & 16 \\
\hline $\begin{array}{l}\text { Rowland } \\
\text { Clark }\end{array}$ & 11.1 & $5.5 \mathrm{~mm}$ & 172 & 9 & 36 & 46 & 8 & 1 \\
\hline $\begin{array}{l}\text { Adair (3GA1) } \\
\text { Late Caddo }\end{array}$ & 11.0 & 6.0 & 77 & 12 & 39 & 39 & 10 & 0 \\
\hline $\begin{array}{l}\text { Hedges ( } 3 \text { HS } \\
\text { Late Caddo }\end{array}$ & 60) & 5.9 & 26 & 15 & 27 & 50 & 8 & 0 \\
\hline $\begin{array}{l}\text { Standridge ( } 31 \\
\text { A.D. } 1300-14\end{array}$ & $\begin{array}{l}\text { MN53) } \\
50 \\
11.1\end{array}$ & 6.0 & 33 & 12 & 43 & 30 & 9 & 6 \\
\hline
\end{tabular}

\section{REFERENCES CITED}

Fritz, Gayle J.

1992 Archeobotanical Remains from Five Sites on the Red River in Northeast Texas. MS on file, Texas Historical Commission, Austin. 